\title{
Periodontal information on the world-wide web
}

\author{
The nature and quality of periodontal related patient information on the world-wide web
}

\section{G. Chestnutt Br Dent J 2002; 193: 657-659}

Background

The internet provides a potentially invaluable source of health education material for members of the public.

\section{Objectives}

This study aimed to investigate the nature and quality of periodontal-related patient information on the world-wide web.

\section{Methods}

The search term " "gum disease" AND "patient information" , was entered into three common internet search engines. The top 50 sites identified by each search engine were downloaded and assessed for authorship, content, accord with accepted dental practice, currency, and references to peer reviewed data.

\section{Results}

The search term returned in excess of 3,615 hits across the three search engines. Of the top 150 identified, 68 were deemed relevant to patients seeking information on periodontal disease. Of these, 17 were duplicated either within or between search engines, therefore the following analysis is based on the remaining 51 sites. The majority of sites (25) were authored by individual dental practitioners, with sites originating in the USA predominating (80\%). Information on the aetiology, signs and symptoms, and treatment options for periodontal disease was provided by 38, 38, and 29 sites respectively. This information was deemed in accordance with conventional periodontal practice in 27, 33, and 23 cases respectively. Only seven sites indicated the currency of the information and five provided peer-reviewed references for further reading. The rank order identified by the search engines meant that sites providing comprehensive and authoritative information, were not readily differentiable from sites whose primary objective was promoting attendance at specific dental practices.

\section{Conclusions}

Judicious and active support from professionals is required to aid patients' interpretation of the information identified and to direct them to the most instructive sites.

\section{IN BRIEF}

- The nature and quality of periodontal-related patient information found on the internet is variable in content and quality.

- Commonly used internet search engines do not readily distinguish the 'wheat from the chaff'.

- Clinicians have a responsibility to a) guide patients to appropriate sources of information on the internet; and b) help patients personalise the information gleaned from the internet to their own personal circumstances.

\section{COMMENT}

The world-wide web is at the centre of the current revolution in information technology. Its very nature uniquely gives unrestricted access to vast amounts of information in a wholly unregulated way. This has profound implications for health professionals and a potential democratising effect for patients in obtaining information about their condition. As a force for health education, this potentially has enormous benefits which will ultimately depend on the accessibility and accuracy of this information.

Here Chestnutt objectively assesses the information available on the world-wide web for patients concerning periodontal disease and its treatment. Given the importance of patient motivation and compliance in periodontal treatment, this is obviously an area of potentially considerable importance. The protocol adopted simulates a real patient-centred search for information using three search engines. It will not be a great surprise to any who have search the world-wide web for all sorts of information that firstly the search produces a very large number of 'hits', in this case over 3,615 and of these approximately $50 \%$ of the top 150 were relevant to the nature of the search. It was encouraging to find that most of the relevant sites contained information that was assessed as 'in accordance with conventional periodontal practice'. It also underlines one of the limitations of such a search engine driven requests for information in that the ranking of sites does not reflect the criteria that dental professionals might use to rank the value and accuracy of sites. Most particularly the absence of consensus and peer review for most sites is possibly a severe limitation on their value.

The study raises a number of issues which need to be looked at further in the future. Firstly, it would be interesting to know the relative credence given by patients to information they obtained from such web sites. Secondly, health professionals need to familiarise themselves with the sort of information available on the web and to be in a position to recommend good sites for patient information. Finally, it might be useful for learned societies to consider the development of formal guidelines for accreditation of web sites to facilitate the patient's ability to obtain high quality, useful information.

Francis J. Hughes, Professor of Periodontology, Barts and The London,

Queen Mary's School of Medicine and Dentistry 